LA-UR- $98-1460$

Approved for public release: distribution is unlimited.

Time Reversal Tests in Polarized Neutron Reactions

Author(s):

J. David Bowman, P-23

Submitted to:

DOE Office of Scientific and Technical Information (OSTI)
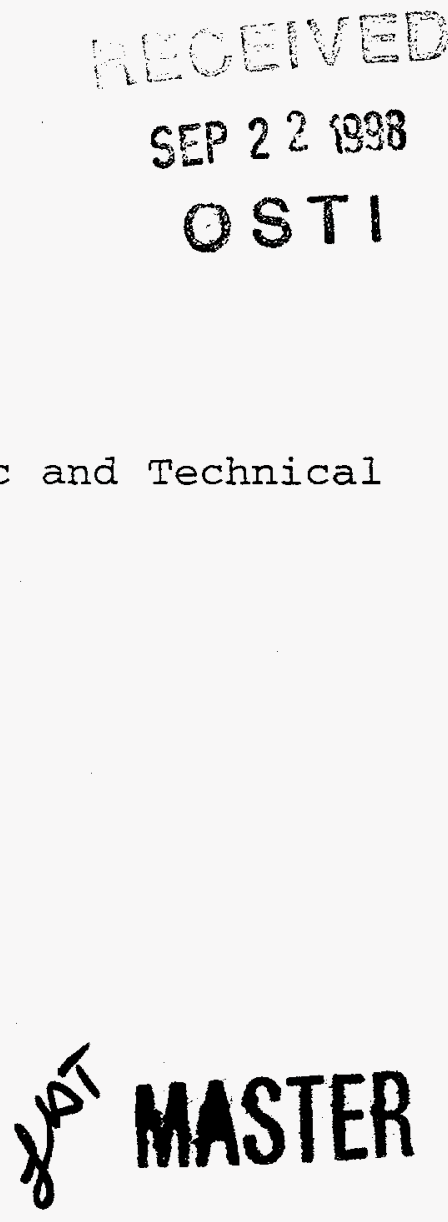

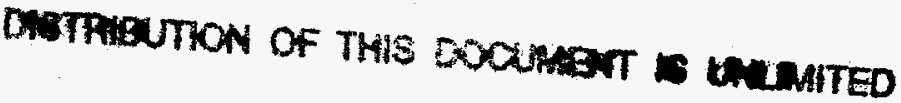

\section{Los Alamos}

NATIONALLABORATORY

Los Alamos National Laboratory, an affirmative action/equal opportunity employer, is operated by the University of California for the U.S. Department of Energy under contract W-7405-ENG-36. By acceptance of this article, the publisher recognizes that the U.S.

Government retains a nonexclusive, royalty-free license to publish or reproduce the published form of this contribution, or to aliow others to do so, for U.S. Government purposes. Los Alamos National Laboratory requests that the publisher identify this article as work performed under the auspices of the U.S. Department of Energy. The Los Alamos National Laboratory strongly supports academic freedom and a researcher's right to publish; as an institution, however, the Laboratory coes not endorse the viewpoint of a publication or guarantee its technical correctness. 


\section{DISCLAIMER}

This report was prepared as an account of work sponsored by an agency of the United States Government. Neither the United States Government nor any agency thereof, nor any of their employees, makes any warranty, express or implied, or assumes any legal liability or responsibility for the accuracy, completeness, or usefulness of any information, apparatus, product, or process disclosed, or represents that its use would not infringe privately owned rights. Reference herein to any specific commercial product, process, or service by trade name, trademark, manufacturer, or otherwise does not necessarily constitute or imply its endorsement, recommendation, or favoring by the United States Government or any agency thereof. The views and opinions of authors expressed herein do not necessarily state or reflect those of the United States Government or any agency thereof. 


\section{DISCLAIMER}

Portions of this document may be illegible in electronic image products. Images are produced from the best available original document. 
Time Reversal Tests in Polarized Neutron Reactions

Authors for Project 95030

Koichiro Asahi (Tokyo Institute of Technology), J. David Bowman* (P-23), Bret Crawford (Duke University), Paul J. Delheij (TRIUMF), Michell Espy (P-21), Tomohito Haseyama (Kyoto University), Shigeru Ishimoto (KEK), Gordon Jones (NIST), Chris Keith (Indiana University), Jim Knudson (L-7), Mark Leuschner (UNH), Akira Masaike (Kyoto University), Yasuhiro Masuda (KEK ), Yasuyuki Matsuda (Kyoto University), Gary E. Mitchell (N.C. State University), Kimio Morimoto (KEK), Seppo I. Penttila (P23), Vance Pomeroy (UNH), Hans Postma (Tech. University of Delft), Dennis Rich (Indiana University), Nathan R. Roberson (Duke University), Kenji Sakai (Tokyo Institute of Technology), Susan J. Seestrom (P-23), Eduard Sharapov (JINR Dubna), Douglas Smith (P-23), Mike Snow (Indiana University), Dave Swenson (Varian), Allan Thompson (NIST), Dale Tupa (P-25), Yi-Fen Yen (Stanford University), Vincent W. Yuan (P-23) 


\title{
Time Reversal Tests in Polarized Neutron Reactions
}

Koichiro Asahi (Tokyo Institute of Technology), J. David Bowman* (P-23), Bret Crawford (Duke University), Paul J. Delheij (TRIUMF), Michell Espy (P-21), Tomohito Haseyama (Kyoto University), Shigeru Ishimoto (KEK), Gordon Jones (NIST), Chris Keith (Indiana University), Jim Knudson (L-7), Mark Leuschner (UNH), Akira Masaike (Kyoto University), Yasuhiro Masuda (KEK), Yasuyuki Matsuda (Kyoto University), Gary E. Mitchell (N.C. State University), Kimio Morimoto (KEK), Seppo I. Penttila (P23), Vance Pomeroy (UNH), Hans Postma (Tech. University of Delft), Dennis Rich (Indiana University), Nathan R. Roberson (Duke University), Kenji Sakai (Tokyo Institute of Technology), Susan J. Seestrom (P-23), Eduard Sharapov (JINR Dubna), Douglas Smith (P-23), Mike Snow (Indiana University), Dave Swenson (Varian), Allan Thompson

(NIST), Dale Tupa (P-25), Yi-Fen Yen (Stanford University), Vincent W. Yuan (P-23)

\begin{abstract}
This is the final report of a three-year, Laboratory-Directed Research and Development (LDRD) project at the Los Alamos National Laboratory (LANL). In recent years the nuclear weak interaction has been studied in the compound nucleus via parity violation. The observed parity-violating effects are strongly enhanced by nuclear structure. The predictions are that the interaction of polarized neutrons with polarized nuclear targets could be also used to perform sensitive tests of time-reversal-violation because of the nuclear enhancements. We have designed experiments to search for timereversal violation in neutron-nucleus interactions. We have also developed techniques to polarize neutrons with laser-polarized ${ }^{3} \mathrm{He}$ gas targets. Using the polarized ${ }^{3} \mathrm{He}$ neutron spin filter, we have performed two experiments at LANSCE: an absolute neutron beam polarization measurement with an accuracy of $0.2-0.3 \%$ and a neutron spin-rotation measurement on a ${ }^{139} \mathrm{La}$ sample.
\end{abstract}

\section{Background and Research Objectives}

One distinct feature of nature is its symmetry. Two important discrete symmetries in nature are space reflection or parity $(\mathrm{P})$ and time reversal $(\mathrm{T})$. Recently, physicists have focused on small symmetry-breaking $P$ and $T$ effects in order to learn more about underlying interactions. Parity violation (PV), which is caused by the weak interaction between fundamental particles, is extensively studied in different systems. The best known reaction mitigated by the weak interaction is beta decay. We have investigated PV in neutron-nucleus interactions at Los Alamos Neutron Scattering Center (LANSCE) [1,2].

As a result of this effort we have measured numerous PV effects in neutron $p$-wave resonances. Some of the observed effects are as large as few per cent.

*Principal Investigator, email: bowman@lanl.gov 
In contrast to the PV phenomenon, we do not know much about the violation of time reversal invariance (TRD). To date there has been no direct evidence of TRI violation. Considerable indirect evidence exists for such a violation through $\mathrm{CP}$ violation in the neutral kaon decay (charge conjugation (C), is the third symmetry of nature). Because we strongly believe that our world is invariant under the combination of the three discrete symmetries CPT, we infer that TRI is violated in the neutral kaon decay. With the study of TRI we can challenge current theories of fundamental physics and we can also shed light on our understanding of the creation of the universe. The origin of the cosmological asymmetry observed in the ratio of baryons to antibaryons is attributed to $\mathrm{CP}$ violation i.e. the violation of TRI.

TRI violation in nuclei is due to a TRI-violating component of the nucleon-nucleon force and it is believed that the observed enhancements in PV effects in $p$-wave resonances can also increase possible TRI violation in neutron-nucleus interactions [3]. Under this LDRD project we proposed to design experiments that would take advantage of the enhancements of symmetry violation in the compound nucleus (CN) to search for a TRI violation. We proposed also to design and build polarized ${ }^{3} \mathrm{He}$ target systems for use as a neutron polarizers and analyzers, to design and build neutron spin transport, and to measure the spin rotation of polarized neutrons passing through a sample of unpolarized ${ }^{139}$ La. This experiment would serve as a preliminary experiment for a test of TRI violation.

\section{Importance to LANL's Science and Technology Base and National R\&D Needs}

Sensitive searches may lead to the observation of TRI violation, which is of great importance for contemporary nuclear and particle physics. This can be seen in the large scale efforts to study CP violation with the B-meson factories that are under construction at SLAC and KEK. One of the Laboratory's strategic goals is to have a world class neutron laboratory in Los Alamos; in addition the Laboratory is strongly pursuing excellence in science. The work of the project described in this report has been done at LANSCE. The objectives of the project address one of the leading problems of nuclear and particle physics and the project has developed state-of-art technology.

\section{Scientific Approach and Accomplishments}

\section{Generality}

The interaction of polarized neutrons with nuclear targets to form compoundnuclear $(\mathrm{CN})$ resonances has developed into a powerful tool for the study of symmetrybreaking components in the nuclear reaction. We have carried out a series of experiments to study PV interaction in neutron resonances $[1,2]$ with the polarized epithermal neutron 
beam at MLNSC. From these data the value of the mean-squared PV mixing matrix element, $M^{2}$, between $\mathrm{CN}$ states is determined using a statistical approach based on the statistical model of the $\mathrm{CN}$. The values of $M$ provide information on the two most important weak meson-nucleon-nucleon coupling constants, $H_{\pi}^{1}$ and $H_{\rho}^{0}$. The success of these PV experiments results from the large enhancements of symmetry violating observables in the $\mathrm{CN}$. These enhancements arise from the small spacing between $\mathrm{CN}$ energy levels and the large ratio of scattering amplitudes between $s$-wave and $p$-wave resonances. The effect of the weak meson-nucleon-nucleon couplings (about $10^{-7}$ ) in the $\mathrm{CN}$ is amplified by as much as $10^{6}$. Because of this enormous enhancement and the availability of intense polarized neutron beams at LANSCE, a large number of nuclear states with PV have been observed.

Stodolsky [3] suggested that the interaction of polarized neutrons with polarized targets can be used to perform sensitive tests of the violation of TRI. He showed that the proposed tests are free from the effects of final state interactions and that they benefit from the same amplifications as the PV effects in $\mathrm{CN}$. The plan of this project was to design and perform preliminary measurements that would take an advantage of these same enhancements to search for a violation of TRI. The observable for the PV studies in the neutron resonances is $\vec{\sigma} \cdot \vec{k}$, where $\vec{\sigma}$ is the neutron spin and $\vec{k}$ is the neutron momentum. There are two observables for a test of TRI violation in the $\mathrm{CN}$ : the first is a parity-odd triple correlation $\vec{\sigma} \cdot \vec{I} \times \vec{k}$ between the neutron spin, nuclear spin of the target $(\vec{I})$ and the neutron momentum, and the second observable is the parity-even five-fold correlation $(\vec{\sigma} \cdot \vec{I} \times \vec{k})(\vec{I} \cdot \vec{k})$. These TRI experiments require a polarized neutron beam, neutron spin analyzer and polarized nuclear target (for the triple correlation) or aligned nuclear target (for the five-fold correlation). The TRI tests in a nucleus are technically more demanding than the PV tests because they involve a polarized (or aligned) nuclear target and a neutron analyzer as well as a polarizer. The PV studies in $\mathrm{CN}$ have shown that the $0.734-\mathrm{eV} p$-wave resonance in ${ }^{139} \mathrm{La}$ is a good candidate for the test of the parity-odd TRI violation. This is because PV in this resonance is large (10\%) [4] and the ${ }^{139} \mathrm{La}$ nucleus can be polarized [5,6]. Also, at the time of the proposal there were great advances in the technology of polarizing low-energy neutron beams using optically-polarized ${ }^{3} \mathrm{He}$ gas targets. Because of the reasons mentioned above we focused on the design of a triple correlation experiment. At Los Alamos we worked on the development of the ${ }^{3} \mathrm{He}$ spin filter technology while our Japanese collaborators worked on polarization of ${ }^{139} \mathrm{La}$.

A search of TRI violation in a polarized neutron transmission experiment through polarized ${ }^{139} \mathrm{La}$ was discussed at Los Alamos [7], in Publication No. 1, and at KEK [5,8]. The proposed experiments are vulnerable to systematic errors and possible fake 
asymmetries, which have been discussed in detail in literature $[3,7,8,9,10]$ and in Publication 1. No triple correlation TRI experiment has so far been done. There are two major difficulties that have to be solved: until recently polarized nuclear targets that have $\mathrm{CN}$ resonances exhibiting a large PV asymmetry have not been available and secondly, it proved difficult to devise an experiment that would isolate the desired effect in the background of other much stronger interactions of the neutron spin.

In last few years our collaborators at KEK and the Kyoto University have achieved substantial, 20\%, polarization of ${ }^{139} \mathrm{La}$ nuclei in lanthanum aluminate crystals [5,6]. In addition, we have also identified a $3.2-\mathrm{eV}$ resonance in the ${ }^{131} \mathrm{Xe}$ isotope that has a longitudinal asymmetry of $3.2 \%$ [11,12]. It may be possible to polarize large quantities of ${ }^{131} \mathrm{Xe}$ using laser techniques [13]. Today there are good prospects that suitable polarized nuclear targets will soon be available.

\section{Optically-polarized ${ }^{3} \mathrm{He}$; a broad-band neutron spin filter}

One of the technical goals of this project was to develop the laser-polarized ${ }^{3} \mathrm{He}$ technology that could be used to polarize low-energy neutrons or analyze neutron spin. Such a technique would be of great importance to many areas of basic and applied research. In recent years, neutron beam polarization by transmission through a sample of laser polarized gaseous ${ }^{3} \mathrm{He}$ has rapidly advanced toward practicality. Two methods have been developed in parallel to polarize ${ }^{3} \mathrm{He}$ nuclei: exchange of polarization from opticallypumped metastable ${ }^{3} \mathrm{He}$ and exchange from optically-pumped $\mathrm{Rb}$. The metastability exchange technique is currently used at Grenoble and Mainz [14]. Since the Rb spinexchange technique was more capable of producing thick (high-pressure) polarized ${ }^{3} \mathrm{He}$ targets, we chose this method. During the project the Rb spin-exchange technique has benefited from industry's production of high powered laser diode arrays (LDA) [15].

Polarized ${ }^{3} \mathrm{He}$ can be used as a nearly perfect spin filter for neutron polarization [16] since ${ }^{3} \mathrm{He}$ has a large neutron absorption cross section for the capture reaction ${ }^{3} \mathrm{He}+\mathrm{n}->\mathrm{p}+\mathrm{t}$ : $\sigma_{a}=\frac{1}{\sqrt{E}} \sqrt{E_{0}} \sigma_{0}$, where $\sigma_{0}=5327$ barn at neutron energy of $E_{0}=0.025 \mathrm{eV}$. The cross section is highly spin dependent due to a broad, unbound resonance in ${ }^{4} \mathrm{He}$ of $J^{\pi}=0^{+}$. In order to conserve angular momentum for this $s$-wave resonance, only the component of neutron spin anti-parallel to the ${ }^{3} \mathrm{He}$ spin is resonantly absorbed. For the component of neutron spin parallel to the ${ }^{3} \mathrm{He}$ spin, only a small potential scattering cross section contributes. When an unpolarized neutron beam passes through polarized ${ }^{3} \mathrm{He}$, beam polarization $P_{n}$ and transmission $T_{n}$ are given by: 


$$
\begin{aligned}
& T_{ \pm}=\exp \left(-n_{3} \sigma_{a} l\left(1 \mp P_{3}\right)\right) \\
& P_{n}=\frac{T_{+}-T_{-}}{T_{+}+T_{-}}=\tanh \left(n_{3} \sigma_{a} l P_{3}\right) \\
& T_{n}=\frac{T_{+}+T_{-}}{2}=\exp \left(-n_{3} \sigma_{a} l\right) \cosh \left(n_{3} \sigma_{a} l P_{3}\right),
\end{aligned}
$$

where $n_{3}$ is the number density of the ${ }^{3} \mathrm{He}, l$ is the length of the ${ }^{3} \mathrm{He}$ polarizer and $P_{3}$ is the polarization of the ${ }^{3} \mathrm{He}$. With simple algebra we get also from eq. (1) for beam polarization

$$
P_{n}^{2}=1-\left(\frac{T_{n}^{0}}{T_{n}}\right)^{2},
$$

here $T_{n}^{0}$ is transmission through the ${ }^{3} \mathrm{He}$ cell when ${ }^{3} \mathrm{He}$ polarization is zero. This relationship shows that to determine the neutron beam polarization we need only to measure the transmission enhancement $\left(T_{n} / T_{n}^{0}\right)$ caused by ${ }^{3} \mathrm{He}$ polarization. We do not need to know the thickness of the ${ }^{3} \mathrm{He}$ or its cross section. Because the neutron- ${ }^{3} \mathrm{He}$ cross section depends upon the neutron energy, the transmission and beam polarization have energy dependencies, which are not indicated in eqs (1) and (2). The ${ }^{3} \mathrm{He}$ spin filter covers a broad energy band as illustrated in Figures 1 and 2 . Figure 1 shows the calculated figureof-merit, $P_{n}^{2} T_{n}$, as a function of neutron energy for three different ${ }^{3} \mathrm{He}$ polarizations of a ${ }^{3} \mathrm{He}$ thickness of $50 \mathrm{~atm} \cdot \mathrm{cm}$. The figure-of-merit defines how long the experiment must run to achieve a certain statistical accuracy. Figure 2 shows $P_{n}, T_{n}$, and $P_{n}^{2} T_{n}$ for the same cell with $50 \%$ polarization. The spin filter cell construction is designed to optimize figureof-merit and includes practical considerations [17] as well. The first experiment using a ${ }^{3} \mathrm{He}$ spin filter employing the Rb spin-exchange method was performed at Los Alamos by Coulter et al. in 1989 [18]. In this experiment an epithermal neutron beam was polarized for a PV measurement. Neutron beam polarization was determined with the accuracy of $10 \%$.

Spin exchange from optically-pumped $\mathrm{Rb}$ vapor via the hyperfine interaction of the $\mathrm{Rb}$ valence electrons with the ${ }^{3} \mathrm{He}$ nucleus was discovered in 1960 by Bouchiat [19]. At that time applications of polarized ${ }^{3} \mathrm{He}$ were limited by a lack of suitable light sources. The polarization of ${ }^{3} \mathrm{He}$ produced by spin exchange evolves with time as

$$
P_{3}=P_{R b} \frac{\gamma_{S E}}{\gamma_{S E}+\Gamma}\left(1-e^{\left(\gamma_{S E}+\Gamma\right) t}\right)
$$

where $P_{\mathrm{Rb}}$ is the steady state $\mathrm{Rb}$ polarization produced by laser pumping, and $\gamma_{S E}=k_{S E}[R b]$ is the rate of spin exchange from the $\mathrm{Rb}$ to the ${ }^{3} \mathrm{He}$ nucleus. $\mathrm{G}$ is the total ${ }^{3} \mathrm{He}$ spin relaxation rate, in general dominated by interactions with paramagnetic 
impurities on the wall of the cell and in the gas. Due to the weakness of spin exchange $\gamma_{S E}=0.06-0.12 \mathrm{~cm}^{3} / \mathrm{s}$, maximum ${ }^{3} \mathrm{He}$ polarization would require both densities of $10^{14}$ $10^{15} \mathrm{Rb} / \mathrm{cm}^{3}$ and ${ }^{3} \mathrm{He}$ relaxation times, $1 / \Gamma$, of many tens of hours [20]. These relaxation times can only be achieved by keeping $\Gamma$ much smaller than the spin exchange rate. This sets strict requirements for the cell material and preparations of the cells [17]. Studies showed that densities of $\mathrm{Rb}$ greater than $10^{14}$ could be effectively polarized when $\mathrm{N}_{2}$ is included to suppress radiation trapping, Corning 1720 alumino-silicate and other low-iron and/or low-helium permeability glasses were used to suppress wall relaxation, and sufficient laser power was absorbed by the $\mathrm{Rb}$ atoms to balance $\mathrm{Rb}$ spin destruction.

At present, the most common lasers in use for $\mathrm{Rb}$ optical pumping in high density ${ }^{3} \mathrm{He}$ targets are high powered laser diode arrays (LDAs) and Ti:sapphire lasers [15]. LDAs dominate current experiments due to price considerations and ease of use compared to Ti:sapphire lasers.

Figure 3 shows a schematic of a spin-exchange optical pumping apparatus. The ${ }^{3} \mathrm{He}$ cell is in an oven that regulates the $\mathrm{Rb}$ density. The ${ }^{3} \mathrm{He}$ is polarized in a 30 -gauss homogeneous magnetic field. Polarization is measured with adiabatic fast passage (AFP) NMR. AFP can also be used to reverse the polarization direction of the filter.

\section{An absolute neutron beam polarization measurement with high accuracy}

The first experiment of this project used a ${ }^{3} \mathrm{He}$ spin filter to measure with high accuracy the absolute neutron beam polarization. The goal of the experiment was to study laser-polarized ${ }^{3} \mathrm{He}$ as a neutron spin filter, the accuracy of the beam polarization measurement, and sources of systematic errors in the beam transmission measurements.

The main systematic error in experiments using polarized low-energy neutrons occurs in the determination of beam polarization. Typically in these experiments the uncertainty is about $1 \%$. With a pulsed neutron source and a ${ }^{3} \mathrm{He}$ spin filter the beam polarization can be measured with the accuracy of $0.1 \%$. This can lead to interesting experiments that probe physics beyond the standard model of the weak interaction such as second-order currents in the free neutron beta decay or violation of TRI in neutron interactions. Using the time-of-flight method the energy of the neutrons from the pulsed source and beam backgrounds can be determined very accurately.

Figure 4 shows main components of the experiment performed on flight path 2 at the Manuel Lujan Neutron Scattering Center (MLNSC). Downstream of the bulk shield a thin ${ }^{3} \mathrm{He}$ ion chamber was used to normalize the beam flux with the accuracy of $10^{-4}[21]$. The neutrons were collimated and transported to the polarized ${ }^{3} \mathrm{He}$ cell located $9 \mathrm{~m}$ from 
the source. The ${ }^{3} \mathrm{He}$ spin filter was mounted on a movable table so that the two ${ }^{3} \mathrm{He}$ cells in the oven, one polarized and one unpolarized, could be alternated in the beam. In addition to neutrons, the beam has a time-dependent gamma ray background. The gamma ray fraction was determined with the absorber technique described in Ref. [22]. Since a beam pulse has a Maxwellian energy distribution, a few very low-energy neutrons can arrive at the detector at the same time as fast neutrons from the next neutron pulse. These slow neutrons were removed from the beam by the ${ }^{3} \mathrm{He}$ gas and a thin $\mathrm{Cd}$ filter. Neutrons were counted with a ${ }^{3} \mathrm{He}$ scintillation detector after traversing the 55 -m flight path [23].

The oven used to control the $\mathrm{Rb}$ density contained two identical ${ }^{3} \mathrm{He}$ cells; one of the cells was continuously polarized perpendicular to the neutron beam with two fibercoupled LDAs. The second cell, containing approximately the same amount of ${ }^{3} \mathrm{He}$ but no $\mathrm{Rb}$, was used as a reference cell. The cells were alternated into the beam every four minutes by changing the position of our target table. Each cell insertion lasted 4800 neutron pulses and formed a complete run in the data analysis. The reference cell was used to normalize the measurement with the polarized cell. A normalization run was performed every four minutes in order to minimize effects of long-term drifts in the apparatus. The wave length, $795 \mathrm{~nm}$, of the lasers was monitored by a spectrometer.

The two cells were each $10 \mathrm{~cm}$ long and $3.5 \mathrm{~cm}$ in diameter. The cylindrical part of the cell was made from reblown Corning 1720 alumino-silicate glass with the wall thickness of $3 \mathrm{~mm}$. At the ends of the cell, the neutron beam windows were made from flat disks, which were attached to the cylindrical cell bodies. Several cells were fabricated and loaded with 50 Torr of $\mathrm{N}_{2}$ and 4-11 atm of ${ }^{3} \mathrm{He}$. The polarization life time in the cells was measured to be $40-50 \mathrm{~h}$ and maximum ${ }^{3} \mathrm{He}$ polarization achieved was $55 \%$. The highest reported ${ }^{3} \mathrm{He}$ polarization to date is $70 \%$ [18].

To determine neutron beam polarization we measured the transmission of the target cell while polarized, $T_{n}^{P}$, and the transmission of the reference cell (always unpolarized), $T_{n}^{R}$.

From eq. (1) we get for the ratio of these transmissions

$$
\frac{T_{n}^{P}}{T_{n}^{R}}=K \cosh \left(n_{3} \sigma_{a} l P_{3}\right)
$$

Both values of the unknown constant $K$ and $\cosh \left(n_{3} \sigma_{a} l P_{3}\right)$, which represents the transmission enhancement caused by the ${ }^{3} \mathrm{He}$ polarization, depend upon the neutron energy. Next we measured the transmission of the target cell while unpolarized, $T_{n}^{0}$.

From this measurement we obtain the polarization independent constant

$$
K=\frac{T_{n}^{0}}{T_{n}^{R}} .
$$


With this method the apparatus needed only to be stable over a period of one run that took about $4 \mathrm{~min}$. The ratio of eqs (4) and (5) gives

$$
\frac{T_{n}^{P}}{T_{n}^{0}}=\cosh \left(n_{3} \sigma_{a} l P_{3}\right) \text {. }
$$

With these three measurements one can determine the argument, $n_{3} \sigma_{a} l P_{3}$, and that can be substituted into eq. (1) to obtain beam polarization, $P_{n}$. This method does not require the knowledge of ${ }^{3} \mathrm{He}$ polarization or thickness of ${ }^{3} \mathrm{He}$. Only three accurate transmission measurements are needed.

Figure 5 shows the preliminary result of the experiment. In Figure 5, beam polarization has been plotted as a function of neutron energy from $20 \mathrm{meV}$ to $10 \mathrm{eV}$. ${ }^{3} \mathrm{He}$ polarization during the measurement shown in Figure 5 was $15 \%$. The measured neutron yield has been corrected for the beam background, detector dead time and length of the flight path. Preliminary results indicate that we have determined beam polarization with the accuracy of $0.2-0.3 \%$. which is the most accurate neutron beam polarization measurement to date. The maximum $\mathrm{He}$ polarization achieved during the experiment was $43 \%$. The experiment is discussed in detail in Publications 2 and 3.

4. Parity-violating neutron spin rotation in the p-wave resonance of ${ }^{139} \mathrm{La}$

According to the optical theorem, PV effects in $\mathrm{CN}$ are caused by the imaginary part of the spin-dependent neutron scattering amplitude. This amplitude was studied in the PV measurements discussed in Section 1. The real part of this PV amplitude induces the neutron spin to rotate about the neutron momentum. Theory predicts a large enhancement of the PV rotation in p-wave resonances. Recent measurements at KEK [5,24] and Gatchina [25] tried to measure the PV spin rotation but the experiments suffered from poor statistics. To develop further the ${ }^{3} \mathrm{He}$ spin-filter techniques, learn more about systematic errors of a TRI experiment, and improve the statistics of the earlier spin-rotation measurements, we proposed to measure the neutron-spin rotation on $0.734-\mathrm{eV} p$-wave resonance of ${ }^{139} \mathrm{La}$ with the high epithermal neutron flux at LANSCE. In our experiment ${ }^{3} \mathrm{He}$ spin filters were used as polarizer and neutron spin analyzer. This was the first experiment where both polarizer and analyzer were ${ }^{3} \mathrm{He}$ spin filters. The experiment was performed in summer 1997. The experimental set-up is shown in Figure 6. The main components of the experiment are: the ${ }^{3} \mathrm{He}$ polarizer, upstream spin-transport magnet, La target (which is inside a superconducting cylinder at zero magnetic field), downstream spin-transport magnet, neutron spin analyzer ( ${ }^{3} \mathrm{He}$ spin filter) and neutron counter (which was a ${ }^{10} \mathrm{~B}$-loaded liquid scintillator [26]). The spin transport magnets, 5-cm La target and the target cryostat were provided by our Japanese collaborators from KEK and Kyoto. To 
reduce the systematic errors over the earlier experiments, the neutron spin was polarized sidewise so that no spin rotation was required during its path through the apparatus. The small vertical component of the neutron spin that is induced by the PV rotation in ${ }^{139} \mathrm{La}$ is held in the downstream vertical field and analyzed by the downstream ${ }^{3} \mathrm{He}$ spin filter and the neutron counter. In order to pick up the PV rotation effect, polarization of the ${ }^{3} \mathrm{He}$ analyzer is reversed every two minutes during the data acquisition. Thus the ratio $\left(N_{+}+N_{-}\right) /$ $\left(N_{+}+N_{-}\right)$, which is the analyzing power $A_{n}=P_{n} \sin (\Theta) \tanh \left(n_{3} \sigma_{a} l P_{3}\right)$, is obtained. Here

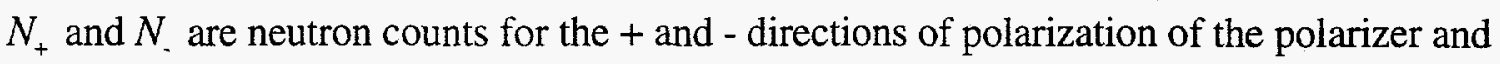
$\Theta$ is a $P V$ rotation angle. The theory of $P V$ in the $C N$ gives a dispersion-like curve for the rotation angle as a function of the incident neutron energy around the $p$-wave resonance. The dispersion curve is determined by the Doppler width, neutron width, and PV matrix element between parity mixing $p$ - and $s$-waves. The expected rotation is about $\partial \phi / \partial z= \pm 0.0026 \mathrm{rad} / \mathrm{cm}$ at $E=E_{p} \pm \Gamma_{p} / 2$, where $\Gamma_{p}$ is the width of the resonance.

The upper part of Figure 7 shows the time-of-flight spectrum around the 0.734-eV $p$-wave resonance. In the lower part of Figure 7 is shown the raw data of the spin rotation. The dispersion nature of the curve is visible. The experiment will be presented in detail in Publication 4.

By polarizing the ${ }^{139} \mathrm{La}$ target, the setup of Figure 6 could serve manifestly as a time-reversal-symmetric apparatus for a measurement of parity-odd TRI correlation, $\vec{\sigma} \cdot \vec{I} \times \vec{k}$.

\section{Future experiments with the ${ }^{3} \mathrm{He}$ spin filter}

a. Precise measurement of $A_{\gamma}$ in reaction $\vec{n}+p \rightarrow d+\gamma$

Although the PV measurements described in Section 1 will provide new constraints [2] on the main meson-nucleon-nucleon coupling constants, $H_{\pi}^{1}$ and $H_{\rho}^{0}$, more precise values of the coupling constants are required to improve the theory of nuclear weak interaction. Also, there exists a controversy around the pion-nucleon-nucleon coupling, $H_{\pi}^{1}$, raised by the ${ }^{18} \mathrm{~F}$ circular-polarization [27] and ${ }^{133} \mathrm{Cs}$ anapole-moment [28] results and their interpretations. Therefore, we have proposed an unambiguous measurement of $H_{\pi}^{1}$ to settle the controversy [29]. The experiment will measure the parity-violating gamma asymmetry, $A_{\gamma}$, in the capture of polarized cold neutrons by parahydrogen in the reaction $\vec{n}+p \rightarrow d+\gamma$. The experiment requires small systematic errors compared to the statistical uncertainty of $0.5 \times 10^{-8}$ that is required to determine $H_{\pi}^{1}$ with an uncertainty of 
$1.0 \times 10^{-7} . H_{\pi}^{1}$ and $H_{\rho}^{0}$ are important because they contribute most of PV phenomena in nuclei.

In this experiment cold neutrons will be polarized with a ${ }^{3} \mathrm{He}$ spin filter of $10 \mathrm{~cm}$ in diameter. With the ${ }^{3} \mathrm{He}$ spin filter the systematic errors of the experiment can be monitored during every neutron pulse. The construction of the ${ }^{3} \mathrm{He}$ spin filter will be a challenge since it will be larger than any previous high-polarization $(50-60 \%){ }^{3} \mathrm{He}$ spin filter. The accurate knowledge of beam polarization is not important for this experiment.

\section{b. Free neutron beta decay}

Neutron decay, $n \rightarrow p+e^{-}+\bar{v}_{e}$, involves solely the first quark generation, $\mathrm{u}$ and

d. The observables in neutron decay depend upon the semileptonic weak coupling constants. A precise knowledge of these coupling constants probes the structure of the electroweak standard model (SM) at low energies. In the SM only two parameters are needed to describe free neutron decay, $\lambda=g_{A} / g_{V}$, the ratio of the axial vector and vector coupling constants, and $V_{u d}$, the first entry in the quark mixing matrix. In neutron decay five observables ( $a, b, A, B$ and D) are accessible to experiments, and together with data from particle and nuclear physics, many tests of SM become possible [30]. Furthermore, all semileptonic cross-sections used in cosmology, astrophysics and particle physics are based on neutron decay data. In particular, free neutron decay measurements are the only measurements precisely determining $g_{\mathrm{A}}$.

At present, the situation is not satisfactory with the neutron decay data base [31]. In some cases, disagreements are interpreted as possible signatures of the existence of right-handed currents in the weak interaction. The largest systematic error in determining the neutron spin correlation coefficients ( $A$ and $B$ ) of free neutron decay is an uncertainty of neutron polarization. Accuracy of the determination is typically around one per cent [31,32]. The use of the ${ }^{3} \mathrm{He}$ spin filter allows beam polarization measurements with the accuracy of $0.1 \%$ and can thus lead to the interesting possibilities to study free neutron decay with a new level of accuracy [33].

In addition to the possibility of performing accurate beam polarization measurements, the ${ }^{3} \mathrm{He}$ spin filter has several important features, which help to improve the precision of these measurements. The acceptance angle of the ${ }^{3} \mathrm{He}$ spin filter is larger than the acceptance angle of super mirrors. The capture of neutrons by ${ }^{3} \mathrm{He}$ does not create gamma rays, which is important for background sensitive experiments. Neutron spin can be reversed by flipping the polarization direction of the ${ }^{3} \mathrm{He}$ spin filter without changing any magnetic fields. This gives an extra method to control systematic errors related to the spin. As explained in Section 2, beam polarization measurements do not require a spin analyzer, 
only careful transmission measurements are needed. Because of the transmission principle of the ${ }^{3} \mathrm{He}$ spin filter the experimental apparatus is simpie, with no reflected beams.

\section{Publications}

1. Bowman, J. D. and Masuda, Y., "Time Reversal Tests in Neutron-Spin Optics," to be published in Phys. Rev. C (1998).

2. Haseyama, T., "Polarized ${ }^{3} \mathrm{He}$ for the Investigation of Fundamental Symmetries with Polarized Neutrons at Los Alamos Laboratory," Master Thesis, Kyoto University, 54 pages, (1996) not published.

3. Penttila, S. I., Bowman, J. D., et al., "An absolute neutron beam polarization measurement with high accuracy using optically-polarized ${ }^{3} \mathrm{He}$," to be published in Nucl. Instrum. Methods (1998).

4. Haseyama, T., "Parity Violating Neutron Spin Rotation in the $p$-wave Resonance of the Neutron-Nucleus Interaction," Ph.D. Thesis, Kyoto University (1998), not published.

\section{References}

[1] Bowman, J. D., Garvey, G. T., Johnson, M. B. and Mitchell, G. E., "Recent Advantages in the Study of Parity Violation in the Compound Nucleus," Ann. Rev. Nucl. Sci. 43, 829-881 (1993).

[2] Bowman, J. D., "Experiments on Parity Violation in the Compound Nucleus," in Proceedings of Parity and Time Reversal Violation in Compound Nuclear States and Related Topics, Auerbach, N. and Bowman, J. D. eds. (World Scientific, 1996) p. 65-81.

[3] Stodolsky, L., "Novel Time-reversal Tests in Low-energy Neutron Propagation," Phys. Lett. 172B, 5 (1986).

[4] Yuan, V. W., Bowman, C. D., Bowman, J. D. et al., "Parity nonconservation in polarized-neutron transmission through ${ }^{139} \mathrm{La}$," Phys. Rev. C 44, 2187 (1991).

[5] Masuda, Y. et al. , "Space time symmetry violation in low energy neutron scattering," in Proceedings of Weak and Electromagnetic Interactions in Nuclei," Ejiri, H., Kishimoto, T. and Sato, T. eds. (World Scientific, 1995) p. 58-67.

[6] Maekawa, T. et al. , "A large nuclear polarization of ${ }^{139} \mathrm{La}$ in $\mathrm{Nd}^{3+}: \mathrm{LaAlO}_{3}$ for testing the time reversal invariance," Nucl. Instrum. Methods, A366, 115-119 (1995).

[7] Bowman, J. D., "Tests of Time-Reversal Symmetry using Epithermal Neutrons," in Proceedings of Tests of Time Reversal Invariance in Neutron Physics, Roberson, $N$. R., Gould, C. R. and Bowman, J. D. eds., (World Scientific, 1987) p. 121-129.

[8] Masuda, Y., "T-Violation in Neutron Nucleus Interaction," in Proceedings of Parity and Time Reversal Violation in Compound Nuclear States and Related Topics, Auerbach, N. and Bowman, J. D. eds. (World Scientific, 1996) p. 83-97. 
[9] Lamoreaux, S. and Golub, R., "General analysis for experimental studies of timereversal-violating effects in slow neutron propagation through polarized matter," Phys. Rev. D 50, 5632-5638 (1994).

[10] Skoy, V. R., “Analysis for an experimental study of time-reversal-violating effects in polarized neutron propagation through a polarized target," Phys. Rev. D 53, 40704073 (1996).

[11] Szymanski, J. J., Snow, W. M, Bowman, J. D. et al., "Observation of a large parity nonconserving analyzing power in Xe," Phys. Rev. C 53, R2576-R2580 (1996).

[12] Skoy, V. R. et al., "The isotopic identification of the parity-violating neutron p-wave resonance at energy $\mathrm{E}_{0}=3.2 \mathrm{eV}$ in Xe," Phys. Rev. C 53, R2576-R2575 (1996).

[13] Cates, G. D. et al., "Laser Production of Large Nuclear-Spin Polarization in Frozen Xenon," Phys. Rev. Lett. 65, 2591-2594 (1990).

[14] Surkau, R. et al. . "Realization of a broad band neutron spin filter with compressed, polarized ${ }^{3} \mathrm{He}$ gas." Nucl. Instrum. Methods, A384, 444-450 (1997).

[15] Cummings, W. J. et al., "Optical Pumping of Rb Vapor using High Power GaAlAs Diode Laser Arrays," Phys. Rev. A 51, 4842 (1995).

[16] Pasell, L. and Schermer, R. I., "Measurement of the Spin Dependence of the ${ }^{3} \mathrm{He}(\mathrm{n}, \mathrm{p}) \mathrm{T}$ Reaction and of the Nuclear Susceptibility of Adsorbed ${ }^{3} \mathrm{He}$," Phys. Rev. 150, 146 (1966).

[17] Chupp, T. E. et al., "Progress Toward a Spin Exchange Pumped ${ }^{3} \mathrm{He}$ Neutron Spin Filter," J. Neutron Res. 5, 11 (1995).

[18] Coulter, K. P., Chupp, T. E., McDonald, A. B., Bowman, C. D., Bowman, J. D. et al., "Neutron Polarization with a Polarized ${ }^{3} \mathrm{He}$ Spin Filter," Nucl. Instrum Methods, A288, 463 (1990); Larson, B et al., "Optical pumping of Rb in the presence of high-pressure ${ }^{3}$ He buffer gas," Phys. Rev. A 44, 3108 (1991).

[19] Bouchiat, M., Carver, T. and Varnum, C., Phys. Rev. Lett. 5, 373 (1960).

[20] Fitzsimmons, W., Tankersly, L. and Walters, G., "Nature of Surface-Induced Nuclear-Spin Relaxation of Gaseous ${ }^{3} \mathrm{He}$," Phys. Rev. 179, 156 (1969).

[21] Szymanski, J. J., Bowman, J. D. et al., "Ion chamber system for neutron flux measurements," Nucl. Instrum. Methods, A 340, 564-571 (1994).

[22] Yen, Yi-Fen, Bowman, J. D. et al., "Determination of the gamma-ray background in a pulsed epithermal neutron beam," accepted for publication in Nucl. Instrum Methods (1997).

[23] Keith, C., Bowman, J. D. et al., "Measurement of the neutron total cross section of ${ }^{3} \mathrm{He}$ in the energy range of $0.1-500 \mathrm{eV}$," forth coming publication in Phys. Rev. C (1998).

[24] Sakai, K. et al., "Measurement of P-violating Neutron Spin Rotation in the p-wave Resonance of ${ }^{139} \mathrm{La}, "$ Hyp. Int. 84, 199 (1994). 
[25] Serebrov, A., "Parity non-conserving neutron spin rotation in the vicinity of ${ }^{139} \mathrm{La} \mathrm{p}$ wave Resonance," JETP Lett. 62, 545 (1995).

[26] Yen, Yi-Fen, Bowman, J. D. et al., "500-MHz Neutron Detector," in Proceedings of Time Reversal Invariance and Parity Violation in Neutron Resonances, Gould, C. R., Bowman, J. D. and Popov, Yu. eds. (World Scientific, Singapore, 1994) 210219.

[27] Adelberger, E. and Haxton, W., "Parity Violation in the Nucleon-nucleon Interaction," Ann. Rev. Nucl. Part. Sci. 35, 501 (1985).

[28] Wood, C. S. et al., "Measurement of Parity Nonconservation and an Anapole Moment in Cesium," Science 275, 1759 (1997); Flambaum, V. and Murray, D., "The anapole moment and nucleon weak interactions", Phys. Rev. C 56, 1641 (1997).

[29] Bowman, J. D. et al., "Measurement of the Parity-Violating Gamma Asymmetry $A_{\gamma}$ in the Capture of Polarized Cold Neutrons by Para-Hydrogen, $\vec{n}+p \rightarrow d+\gamma$ ", Research Proposal," not published (1997).

[30] Deutsch, J. et al., in Proceedings of Precision Tests of the Standard Electroweak Model, Langacker, P. ed. (World Scientific, 1995).

[31] Yerozolimsky, B. G., "Free neutron decay," Contemp. Phys. 35, 191-208 (1994).

[32] Abele, H. et al., "A measurement of the beta asymmetry $A$ in the decay of free neutrons," Phys. Letters B 407, 212-218 (1997).

[33] Bowman, D. J., "Experimetal Study of Neutron Beta Decay at LANSCE," seminar talk, not published (1997).

\section{Figure Captions}

Figure 1: Figure-of-merit, $P_{n}^{2} T_{n}$, for a neutron spin filter with helium polarizations of $40 \%$ (solid), 50\% (dotted and dashed), and 60\% (dashed), and with a target thickness of 50 atm. $\mathrm{cm}$.

Figure 2: Neutron transmission, $T_{n}$, (dotted and dashed), polarization, $P_{n}$, (solid), and figure-of-merit, $P_{n}^{2} T_{n}$ (dashed) for a neutron spin filter with $50 \%$ helium polarization and a target thickness of $50 \mathrm{~atm} \cdot \mathrm{cm}$.

Figure 3: Schematic diagram of the ${ }^{3} \mathrm{He}$ spin filter. Shown are the laser, optics, holding field coils, RF coils for NMR measurement of ${ }^{3} \mathrm{He}$ polarization, oven, pick-up coils for NMR and signal processing electronics.

Figure 4: Conceptual setup of the high-accuracy beam polarization experiment at MLNSC.

Figure 5: Neutron beam polarization as a function of neutron energy from $20 \mathrm{meV}$ to 10 $\mathrm{eV}$. The ${ }^{3} \mathrm{He}$ thickness is $50 \mathrm{~atm} \cdot \mathrm{cm}$ and ${ }^{3} \mathrm{He}$ polarization is $15 \%$. 
Figure 6: Schematic diagram of the spin-rotation experiment. Neutrons are polarized with ${ }^{3} \mathrm{He}$ spin filter. The polarized neutrons are transported to the 5-cm-long La target which is inside the superconducting shield at zero magnetic field. The neutron spin is then transported to the analyzer, which is another ${ }^{3} \mathrm{He}$ spin filter, and finally the neutrons are counted by a detector.

Figure 7: Upper part: Time-of flight spectrum of the 0.734-eV p-wave resonance of ${ }^{139} \mathrm{La}$. Lower part: Dispersion curve of neutron spin rotation from the on-line data of the experiment. 


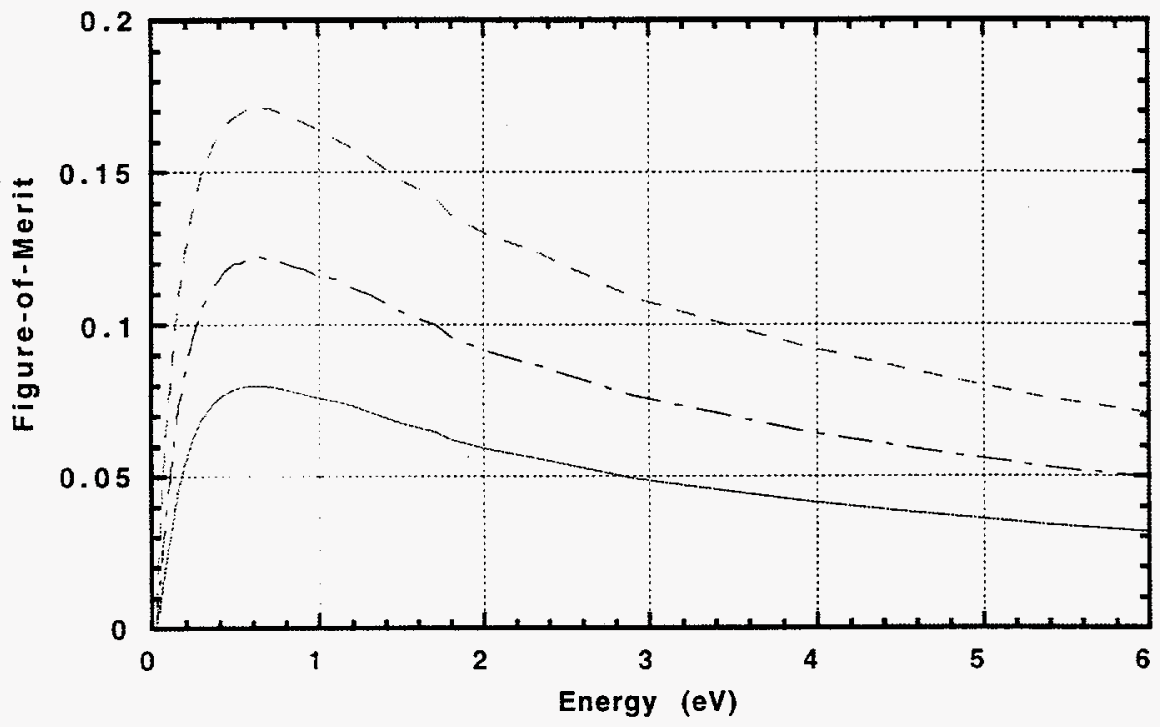

Figure 1 


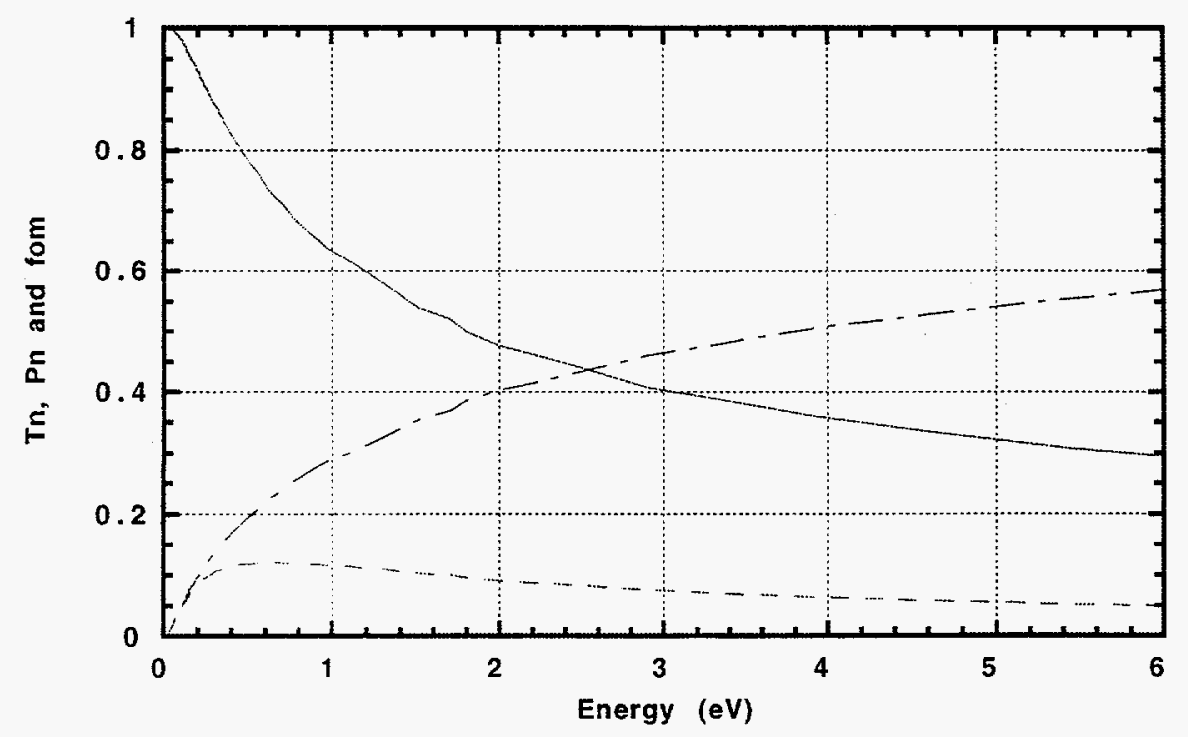

Figure 2 


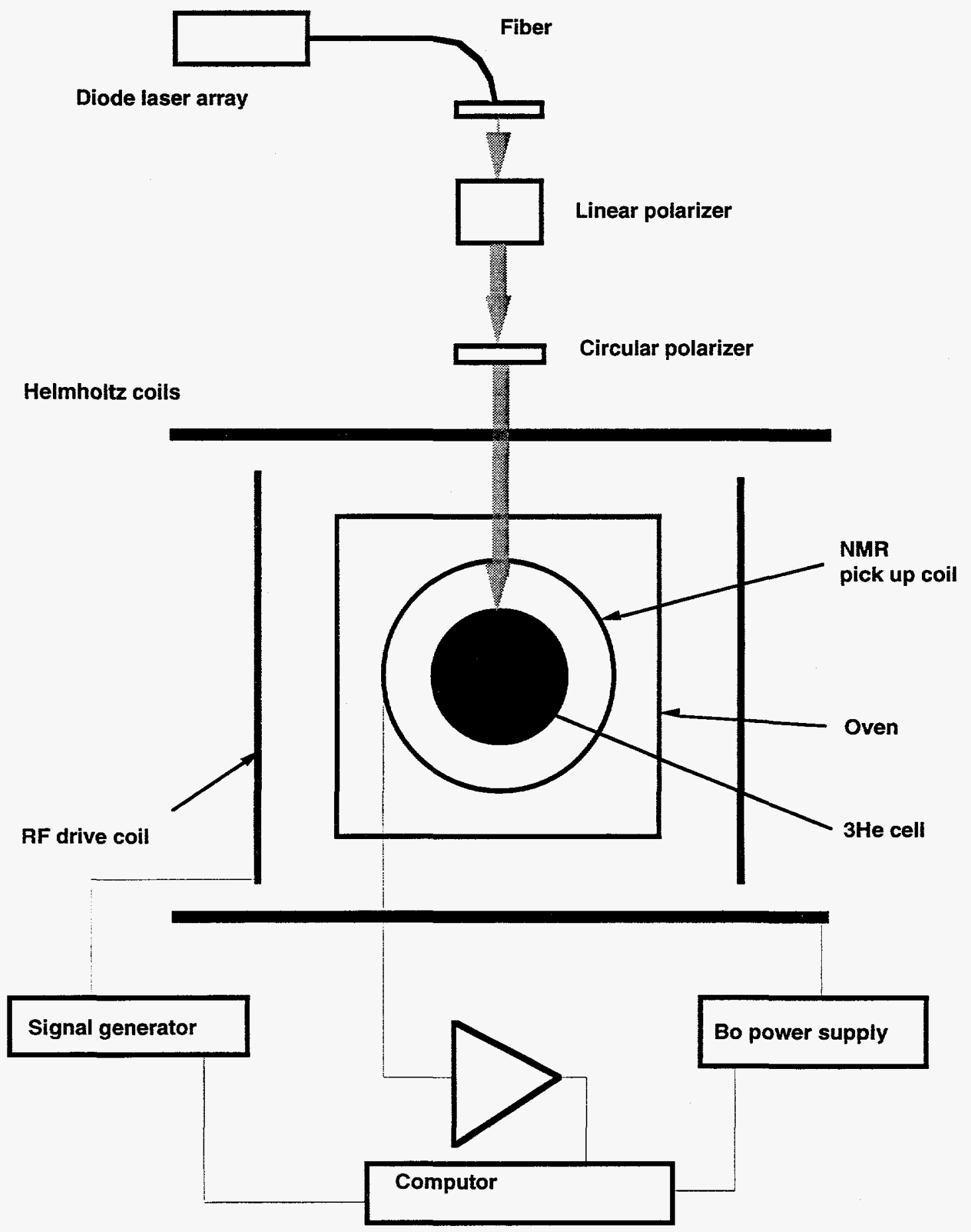

Figure 3 


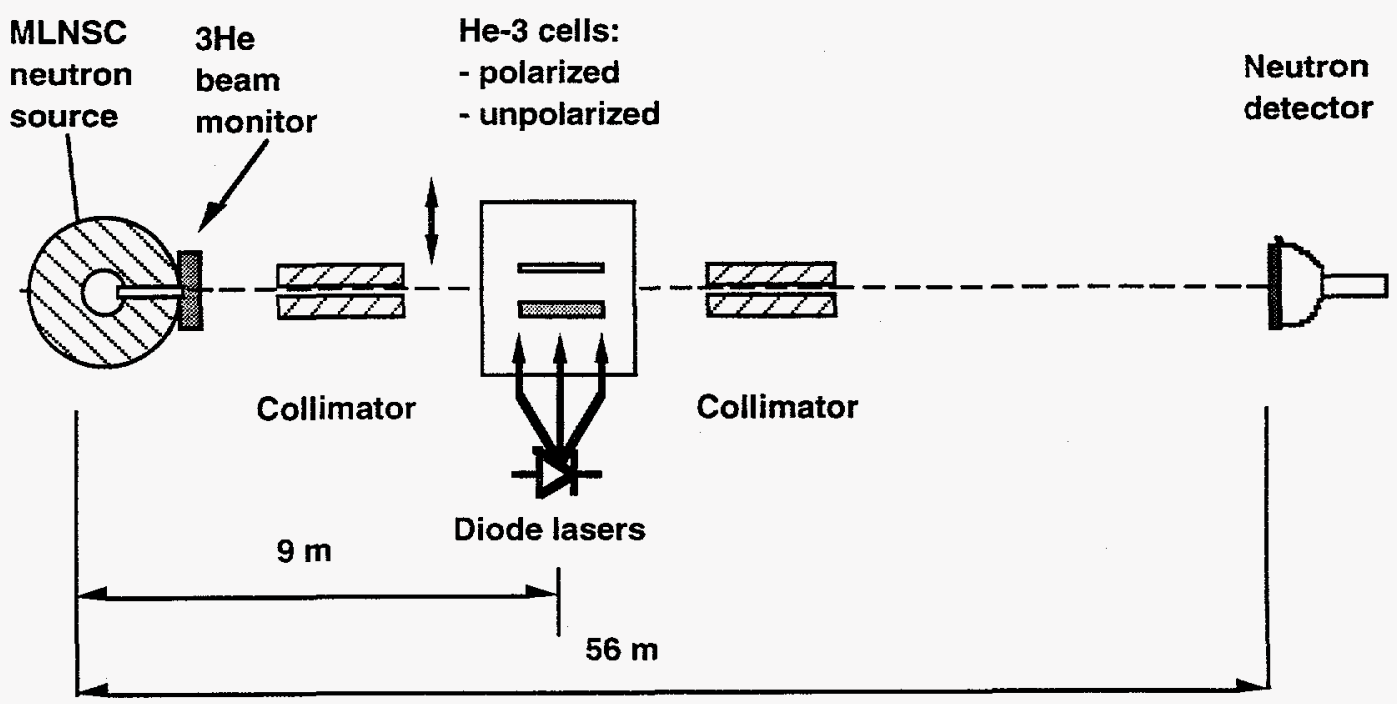

Figure 4 


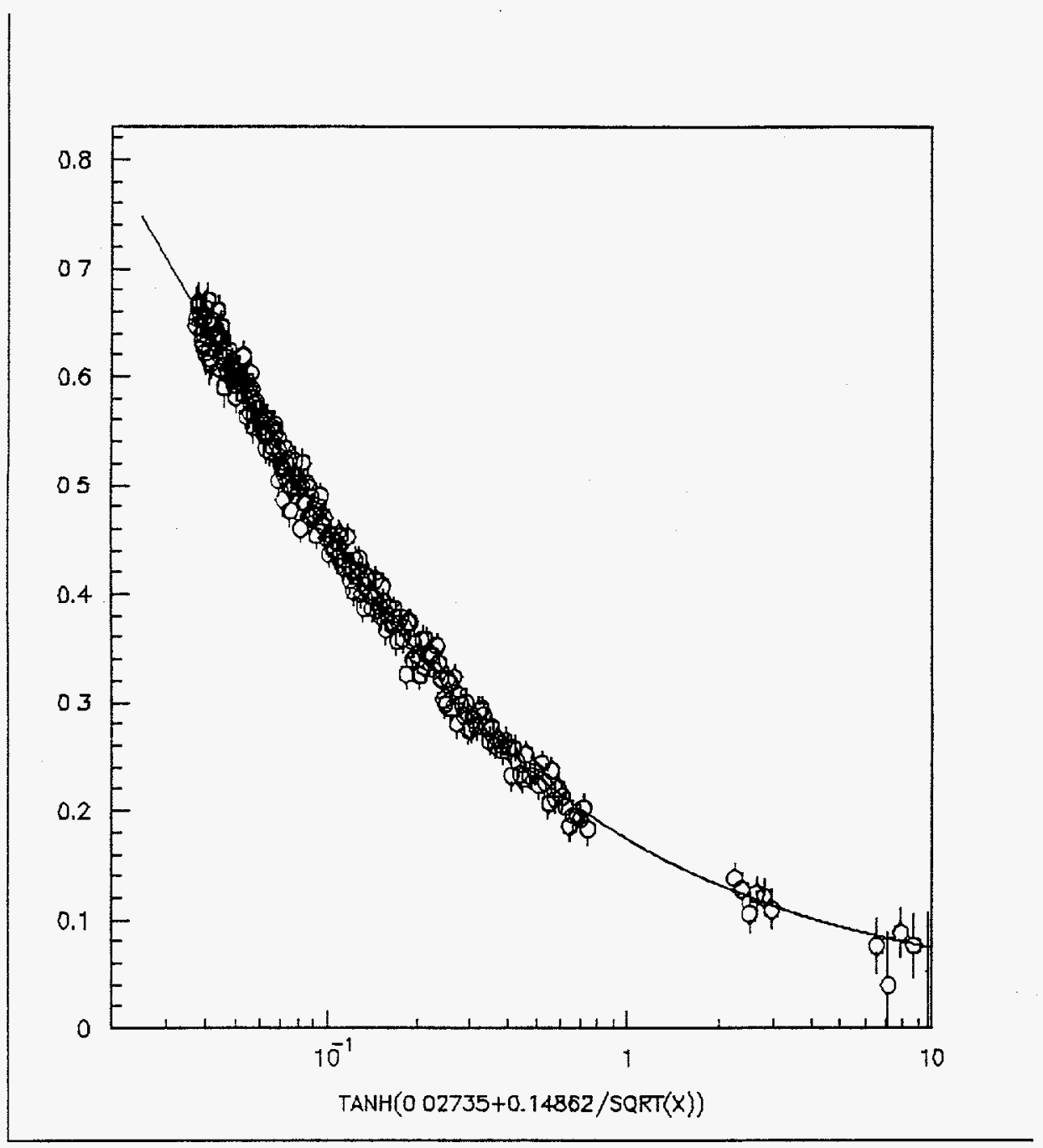

Figure 5 


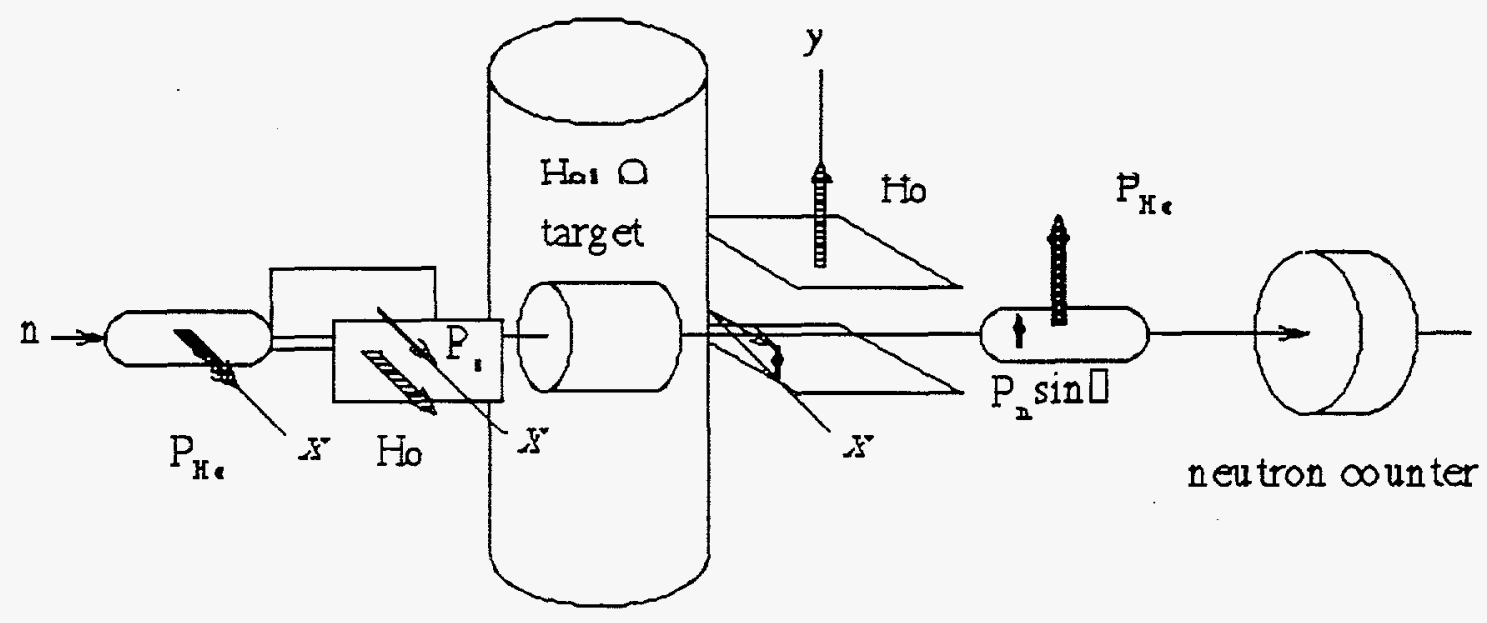

superoonducting cylinder

Figure 6 


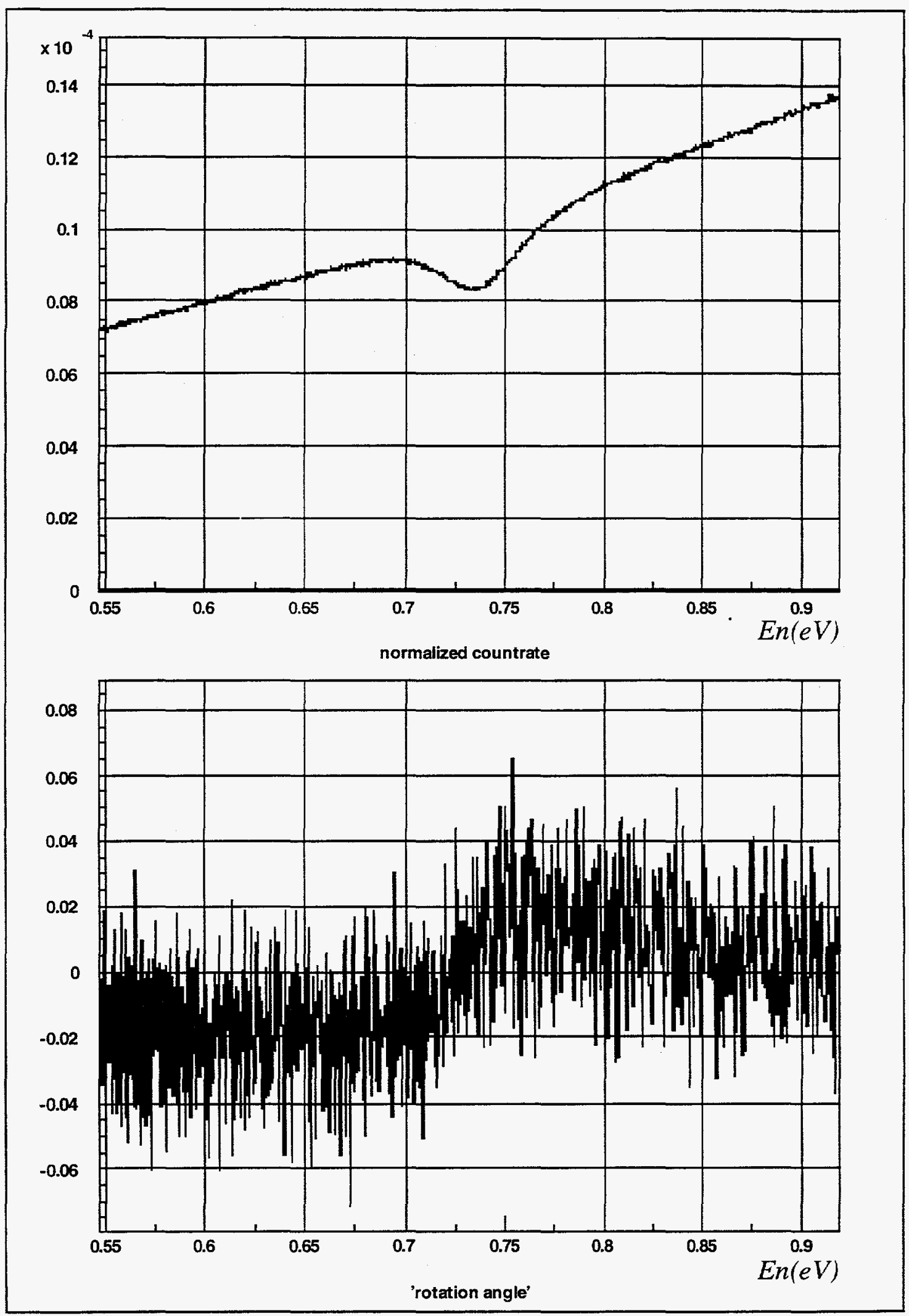

Figure 7 